\title{
Aspects of drying and cracking of geomaterials in energy applications
}

\author{
Bolesław Mielniczuk ${ }^{1}$, Said Moulay El-Youssoufi ${ }^{2}$, and Tomasz Hueckel ${ }^{1, *}$ \\ ${ }^{1}$ Duke University, Durham, USA \\ ${ }^{2}$ LMGC UMR 5508, CNRS-Université de Montpellier, France
}

\begin{abstract}
Because of common exposure to high temperatures and forced ventilation of geomaterials in energy production and storage, their failure mode often involves intense drying and constrained shrinkage associated cracking. Previous experiments show that drying cracking occurs when the materials are completely saturated, practically simultaneously with the onset of air entry and start of desaturation. The paper reports on the adhesion forces evolving in drying granular clusters and their critical value associated with the first air entry and formation of the drying cracks, as well as a critical saturation for meso-scale evaporating several grain cluster models of different porosities.
\end{abstract}

\section{Introduction}

Drying and more generally, penetration of non-wetting fluids into a saturated geomaterial are phenomena that are relevant to several technologies of unconventional energy production/management. These include potential cracking and its prevention in all geologically based barriers subject to nuclear waste heating and ventilation of galleries and buffers, $\mathrm{CO}_{2}$ injection into a saturated rock, drying of pools of liquid residuals of mineral mining.

The phenomena contributing to drying cracking are multiple and of uncertain timing, rate, coupling and scale to be represented in. They include evaporation of surficial water, evaporation of capillary and adsorbed clay water, transport of water and vapor through pore, air entry, displacement of water/air interface, evolution of suction, suction resultant force and surface tension resultant force, and hence of adhesion force, deformation of the pore space/solid skeleton, drying shrinkage, effective stress evolution due to constrained shrinkage, effect of air entry on stress distribution and stress concentration due to air entry, onset and development of drying cracking, 1D, 2D and 3D- evolution of drying crack systems, evolution of permeability in drying crack systems.

It is essential for security of many energy installations to predict the conditions for drying cracking, monitor for their occurrence, possibly to avoid them through either engineering the service loads, building additional or different barriers, or to invent reinforcement of the barrier materials at any scale, from nano-scale to macro-scale.

This paper reports on the multi-physics processes as recently determined in multi-scale experiments at the scale of clusters of several glass grains undergoing drying, and cracking resulting from kinematic constraints imposed externally at a meso-scale. As well documented, drying cracks in macro-scale experiments form nearly simultaneously with soil attaining shrinkage limit and air entry. These two are phenomenological macro-scale thresholds of water content, or pore pressure commonly linked to averaged variables at the laboratory defined experimental conditions. Here, we focus on the air entry conditions at micro-scale of glass grain clusters, which according to our postulate [1] constitute an induced material imperfection, leading to the kinematically constrained drying crack propagation onset.

\section{Materials and methods}

The tests were performed in the apparatus presented in Fig. 1 (see [2] for details). The apparatus is designed to obtain images and measure intergranular forces between two parallel row of grains: lower set sitting on a scale, the upper one hanging from a cantilever fixed to the wall. The two sets are connected by a liquid bridge. The liquid with surface tension of $0.05 \mathrm{~N} / \mathrm{m}$ is distilled water, the glass beads are precision borosilicate spheres, 3.5 or $8 \mathrm{~mm}$ of diameter.

Digital camera continuously recorded the evolution of the projection of the capillary bridge body. To image the moment of rupture, a high-speed digital camera (Vision Research Phantom v12) was employed, with the frame rate of more than 27000 images per second. The separation between the two sets is set by a micrometric screw. The tests reported in this paper were performed by enabling evaporation at a constant relative humidity in the test chamber, at a constant separation.

\footnotetext{
${ }^{*}$ Corresponding author: hueckel@duke.edu
} 


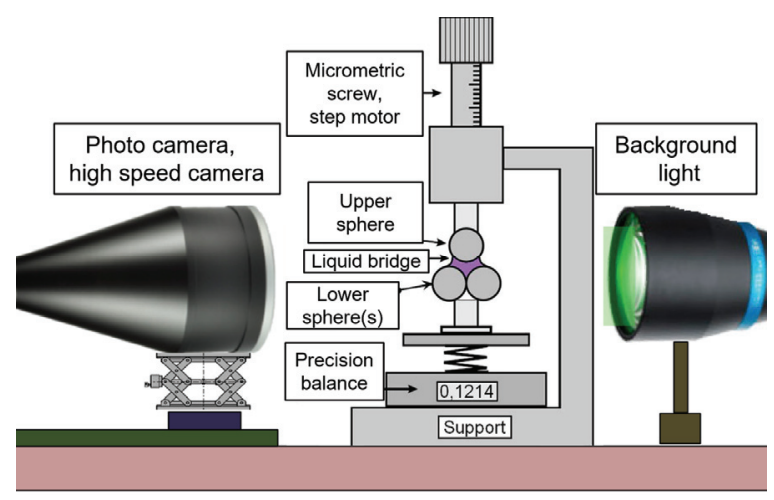

Fig.1 Schematic of the adhesion force apparatus

The objective of the study is to follow the evolution of the wet granular system through the process of drying, by recording the history of the configuration of the gas/liquid interface and simultaneous intergranular force changes, the visible profile interface curvature evolution and whenever possible, the corresponding total capillary (Laplace) pressure, - to any degree of approximation - of 5-grain, 4-grain, 3-grain and 2-grain sub-systems.

\section{Water configuration evolution}

\subsection{Results}

Evaporation of monodisperse systems is analyzed at a mesoscale of a clusters of a few grains connected by a body of water kept in place by capillary forces. The evaporation consists of intercalating phases of a relatively slow and relatively uniform external surfaceevaporation, suddenly transitioning through an almost instantaneous localized air entry. The air entry consists of a rearrangement of water mass, with a completely different interface morphology, followed in most cases, by another phase of slow surface-evaporation. Interestingly, the modes of air entry occur in three different forms, shown in Fig. 2, consisting in: (i) a thinsheet instability (Fig. 2a, b and c) - in the case of twodimensional fragments of the granular systems, (ii) meniscus snap-through (Fig. 2e, f and g) and (iii) formation of a water column and its rupture through pinching (Fig. 2d) - in the case of two-grain bridges. The air entry modes (i) and (ii) develop for systems of any number of grains, except for just two-grain systems. Column pinching (iii) takes place for 2-grains systems, only. The latter mode is discussed in detail by Mielniczuk et al. [3, 4].

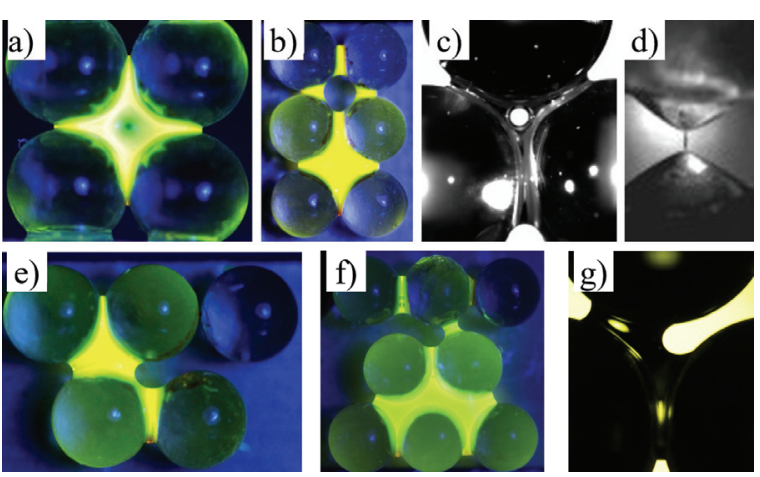

Fig. 2. Three configuration modes of air entry: (a), (b) and (c) thin-sheet instability for 4-, 6- and 3- grain systems; (d) water wire formation and a pinch-off for a 2-grain system; (e), (f) and (g) meniscus snap-through for 5- 8- and 3- grain systems, respectively

The above three modes of evolution of the water body configuration depend on the mode of air entry into the water body. The air entry mode is different for positive (double bowl), negative (or saddle) and zero (flat, at least in one cross-section) Gaussian curvature (GC) points. GC is the product of the two principal curvatures of the water body surface. We perceive the air entry as local instabilities of the external water surface. The instability for postive GC is in a form of bifurcation of a thin-sheet tension with void nucleation and spreading, whereas for negative GC, the instability takes a form of a meniscus snap through. The evolution occurs in both cases very fast, within about $1 / 1000$ second, which is completely out of scale of the evaporation process, hence becoming a fluid dynamic instability phenomenon. More details are presented in [5]. Currently, with the imaging techniques employed, we are not able to provide a numerical value of the GC at air entry, nor we are in the position to advance a hypothesis about quantitative criteria for the air entry mode. Further studies are needed to determine the quantitative and physical conditions for the development of these instability modes.

For largest grain structures of 5-grains investigated in this study, it has been seen that the mode of air entry depends on the separations between the grains, which may be seen as a representation of porosity of the macroscale granular medium. However, in general, the larger the separations, the shorter the time to the first entry and the higher the corresponding cluster saturation. Cluster saturation is defined as a ratio between the current total volume of water and a reference (initial) volume of water filling the space between the grains in the initial configuration. The current total volume of water includes water in isolated small bodies that do not communicate with the "main" body of water contiguous with the stress constrained boundary. 


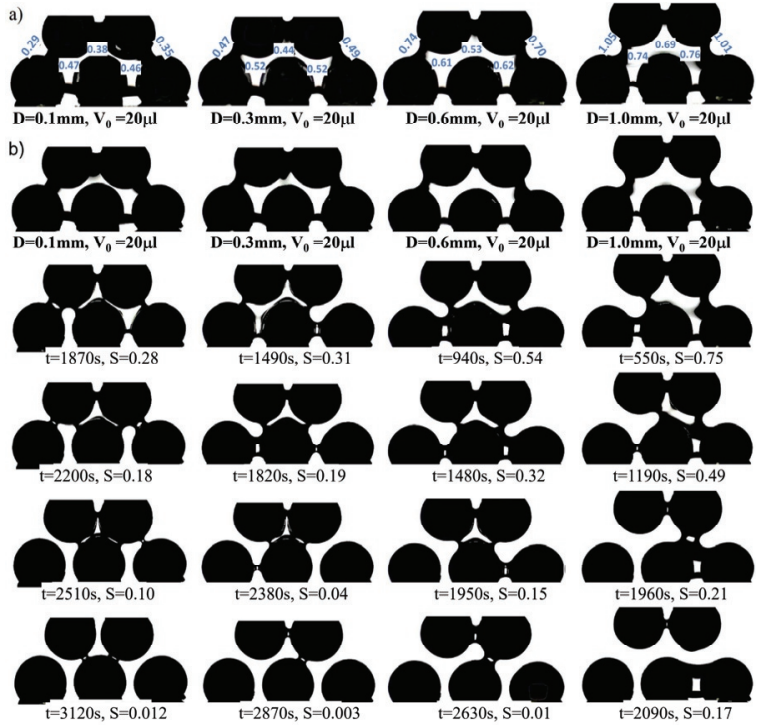

Fig. 3. Five grain capillary water (loose cluster) transitioning through capillary, funicular and pendular configurations: (a) separations between individual grains; (b) changes in configurations for different separations: of $0.1 \mathrm{~mm}$, first column; of $0.3 \mathrm{~mm}$, second column; $0.6 \mathrm{~mm}$, third column; $1 \mathrm{~mm}$, last, fourth column.

Specifically, in loose structures (larger grain separations) the air entry events take place through a meniscus instability (at a negative GC point), see Fig. 3, whereas in tighter structures (smaller grain separations) the AE occurs via thin sheet instabilities at positive GC points see Fig. 4. The localized air entry results into a transition from one configuration to another in an unstable process which involves a fast change in geometry and a rapid displacement of water masses. Separation of grains is kept constant over the entire duration of the tests presented, unless mentioned otherwise. The patterns of the sequences of air entries depend on the separation.

For loose clusters, at the smallest separation of $0.1 \mathrm{~mm}$, the first air entry converts a 5 -grain cluster into a doublet and a 4-grain cluster (Fig. 3). The subsequent air entry yields two doublets, and a 3-grain cluster. The latter one shares two top grains with the doublets. Further, the doublets undergo a pinch-off, while the triplet exhibits another air entry generating two doublets. At all larger separations the patterns are similar, but not identical.

For tight clusters, with zero horizontal gaps between the grains (within the rows, and with a half of the initial water volume compared to the loose systems, Fig. 4, the instabilities present two common features with the loose systems. First, the process consists in: a slow motion of the external boundary at the rate of evaporation, followed by an abrupt, fast and substantial alteration of the boundary, and followed again by a slow motion of the boundaries, until the next instability develops. Second, the sequence of instabilities appears to be sensitive to random imperfections in the symmetry of the system. However, the initial instability for the tight systems has a completely different mechanism, as well as location, from that in loose clusters, and it occurs at one of two points with the double bowl-type (positive $\mathrm{GC}=$ both convex) curvature. a)

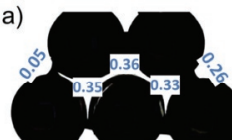
$\mathrm{D}=0.1 \mathrm{~mm}, \mathrm{~V}_{0}=20 \mu \mathrm{l}$

b)

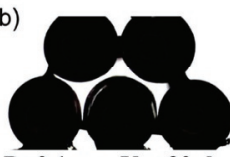

$D=0.1 \mathrm{~mm}, V_{0}=20 \mu \mathrm{l}$

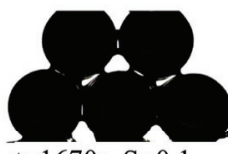

$\mathrm{t}=1670 \mathrm{~s}, \mathrm{~S}=0.1$

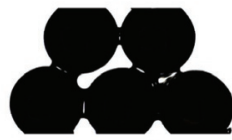

$\mathrm{t}=1930 \mathrm{~s}, \mathrm{~S}=0.035$

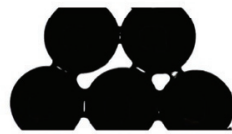

$\mathrm{t}=1970 \mathrm{~s}, \mathrm{~S}=0.028$

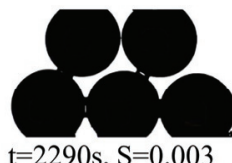

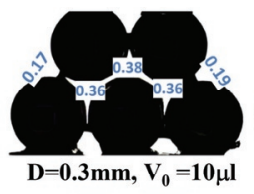
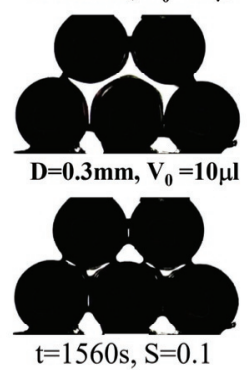

$\mathrm{t}=1750 \mathrm{~s}, \mathrm{~S}=0.04$

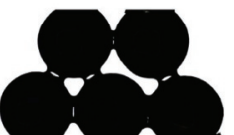

$\mathrm{t}=1800 \mathrm{~s}, \mathrm{~S}=0.032$

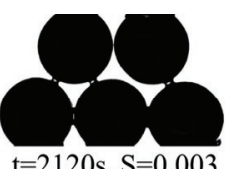

$\mathrm{t}=2120 \mathrm{~s}, \mathrm{~S}=0.003$

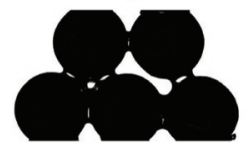

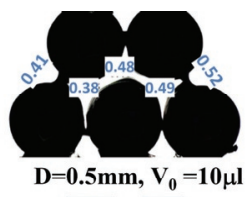
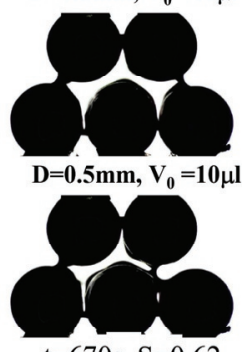

$\mathrm{t}=670 \mathrm{~s}, \mathrm{~S}=0.62$
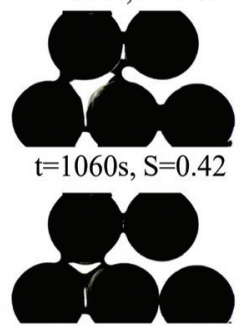

$\mathrm{t}=1560 \mathrm{~s}, \mathrm{~S}=0.19$

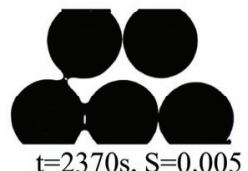

Fig. 4. Five grain capillary water (tight cluster) transitioning through capillary, funicular and pendular configurations: (a) separations between individual grains; (b) changes in configurations for different separations: of $0.1 \mathrm{~mm}$, first column; of $0.3 \mathrm{~mm}$, second column; $0.5 \mathrm{~mm}$, third column;

For the separation between the rows of $0.1 \mathrm{~mm}$ and $0.3 \mathrm{~mm}$ the first instability occurs (after about $30 \mathrm{~min}$. for $0.1 \mathrm{~mm}$ and $26 \mathrm{~min}$ for $0.3 \mathrm{~mm}$ ) through a thinning of water body from the front and back, at the central point of the pore. As the thickness of the water body decreases with the evaporation, it causes the front and the back surfaces to approach, forming effectively a thin-sheet at the point of local symmetry. The thin-sheet then develops an instability in the form of a circular hole, which expands into a curvilinear triangle, and then stabilizes. This form of instability of a thin sheet is well known in thin sheet fluids (see [6,7]). For this central point of the pore, the diameter of an inscribed circle between grains at the equatorial level is $0.363 \mathrm{~mm}$ (or $0.38 \mathrm{~mm}$ ), which effectively is the largest inter-grain gap of the system.

For the largest separation in tight systems, $0.5 \mathrm{~mm}$, the instability mode is of the saddle type. In fact, the side gap for that separation is quite large. Compared to loose systems, except for $\mathrm{D}=0.5 \mathrm{~mm}$, the tight ones develop their first instabilities (air entry) at a more advanced desaturation.

Finally, for the two smallest separations in tight systems, $0.1 \mathrm{~mm}$ and $0.3 \mathrm{~mm}$ after an initial double bowl 
instability, the subsequent mode is that of a saddle meniscus instability, followed again with a double bowl instability at the remaining pore location.

In view of the evolution of the large system of 5 grains into 3- and 2- grain sub-systems, it is interesting to see that the evolution of such smaller but isolated systems follows the same patterns, as shown in an example of 3-grain system in a tight clusters evolving into 3 pendular bridge configuration trough double bowl instability and loose arrangement evolving into two pendular bridges via a saddle instability, as shown in Fig.5 a and b, respectively.

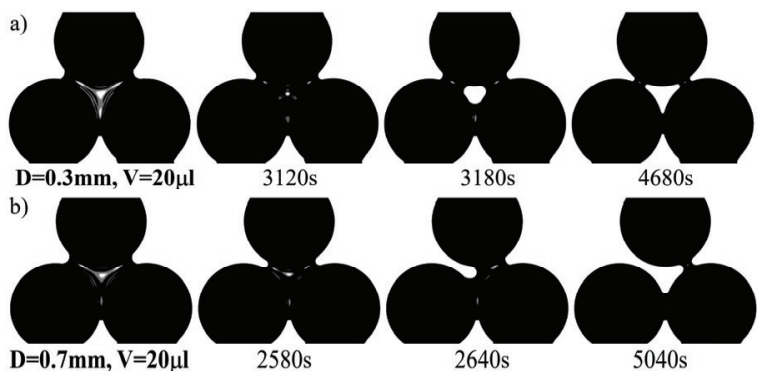

Fig. 5. Three grain capillary water bridge evolution: (a) tight cluster) transitioning to three pendular configurations via double bowl instability; (b) loose cluster transitioning to two pendular configurations via saddle instability

Notably, at a larger scale, such as that modeled in Hale-Shaw apparatuses, the sequence of the air-entries forms a high saturation gradient "drying front" tens to hundred grain diameters thick (see e.g. [15]).

\subsection{Discussion 1}

It is interesting to note that after the first (thin-sheet) instability, the system evolved into a symmetric configuration, reduced to two parallel 3-grain subclusters and one horizontally connected 2-grain bridge for both $0.1 \mathrm{~mm}$ and $0.3 \mathrm{~mm}$ separations. Further developments are those of such sub-systems. For both 3grain sub-clusters, with a newly formed saddle, sidewise meniscus instability occurs, spilling eventually to the neighboring positive GC point. Immediately after, an independent and not- connected thin-sheet instability formed in the remaining minimal positive GC point, for both 0.1 and $0.3 \mathrm{~mm}$. Notably, the cluster saturations are very low in both instances. Growth of the body of air and the previous one eventually leads to formation of a system of pendular bridges between pairs of grains, which finally rupture one after another, after ultimately evaporating a substantial fraction of their starting mass of water. The remaining (often called residual) water forms stress-free water films around the pair-forming grains and evaporates without affecting stress transmission in the system.

One needs to address at this point the fact that since early on, one observes masses of water, often significant, that become isolated from the others, and hence not subject to any pressure gradient flows, and if evaporation conditions stop, behave as an inert water mass, and in analogy to strongly adsorbed water in clays effectively becomes a part of the solid phase (see [8]). These isolated masses of water, while subject to capillary forces, do not participate in any internal (or external) force transition chain. In the context of meso- and macro- scale media it appears more appropriate to refer to total (resultant) intergranular forces acting between the grains across a nominal surface area of a cluster, rather than to pressure (suction) in water bodies as a means to transfer the loads, as they may become separated in the process of drying.

As shown by Hueckel et al. [5], the isolated threegrain clusters exhibit qualitatively the same patterns as three-grain sub-clusters of the five-grain systems discussed above.

\section{Intergranular force evolution}

\subsection{Results}

In parallel to image collection, the tests on 5- grain clusters included the measurements of the intergranular force between the top two grain row and the bottom row, the latter of which was sitting on a scale, following the same procedure described in [2].

The results, presented in Fig. 6, indicate a qualitative similarity to the capillary force evolution for two-grain systems [4] and three- and four-grain systems [5], that exhibit between $30 \%$ to $300 \%$ increase of the initial attractive capillary force to a maximum, followed by a drop to zero of the force at cluster saturation approaching zero.

The maximum force value depends on separation: the larger the separation, the lower and earlier the maximum value. The force shows a series of up to three discontinuities, each of up to $25 \%$ of the maximum force value. The force discontinuities coincide with the instability events, and invariably occur during a decreasing force portion of the process.
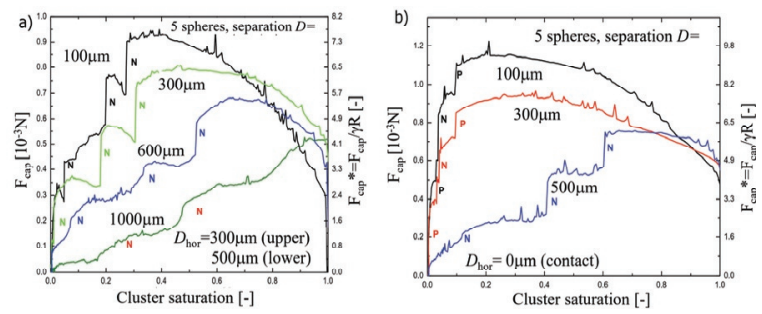

Fig. 6. Resultant capillary force evolution between the top and the bottom rows of grains in 5 - grain clusters as a function of cluster desaturations for different grain separations: (a) loose clusters; (b) tight clusters instability. $\mathrm{N}=$ negative, $\mathrm{P}=$ positive Gaussian curvature point instability (from Hueckel et al., 2019)

For loose systems all discontinuities occur via meniscus instability mechanism only (negative GC), while for tight systems and at smaller separations 100 and $300 \mu \mathrm{m}$, the discontinuity may occur also via thin sheet instability (positive GC), whereas at a larger separation, $1000 \mu \mathrm{m}$ the instabilities take place via meniscus instability. Especially for the loose systems, there is a slight rebound of the force after the drop. 
The resultant intergranular force for 3- and 4- grain systems, obtained by Hueckel et al. [5], show a similar pattern to the 5-grain system. One additional observation suggests that double bowl instability in tight systems coincides with a decreasing intergranular force from the onset of evaporation. In contrast, the saddle instability is associated with an intergranular force initially growing. Notably, all instabilities take place always at a decreasing segment of the intergranular force.

\subsection{Capillary adhesion force at the air entry}

As argued before [5], at least at the micro-scale, the grains of clusters partially submerged in water are attracted via water bridges by two kind of forces: a resultant of water pressure (most often negative) acting during evaporation across a diminishing solid/liquid interface area and resultant surface tension force acting along a decreasing solid/liquid/air contact line length. The order of magnitude of the two resultants that were measured experimentally for two grain bridges, are very close, but their evolution trends are different. Similar conclusions were reached based on their Molecular Dynamics simulations for nano-scale capillary bridges (between two flat solid plates) by Fernandez-Toledano et al. [9]. It was therefore concluded [5], that rather than considering the evolution of an average capillary (Laplace) pressure as a sole variable controlling the cohesion as in unsaturated porous media mechanics (see e.g. $[10,11])$, it is more comprehensive to use the total intergranular capillary force as a variable controlling the cohesion of the evaporating medium at a meso-scale, and reaching critical values at the moment of the first and subsequent air entries.

Fig. 7 shows the intergranular force value at its maximum, and then at an air entry moment for a succession of entry points presented against cluster saturation, for 5 - grain clusters, both tight and loose.

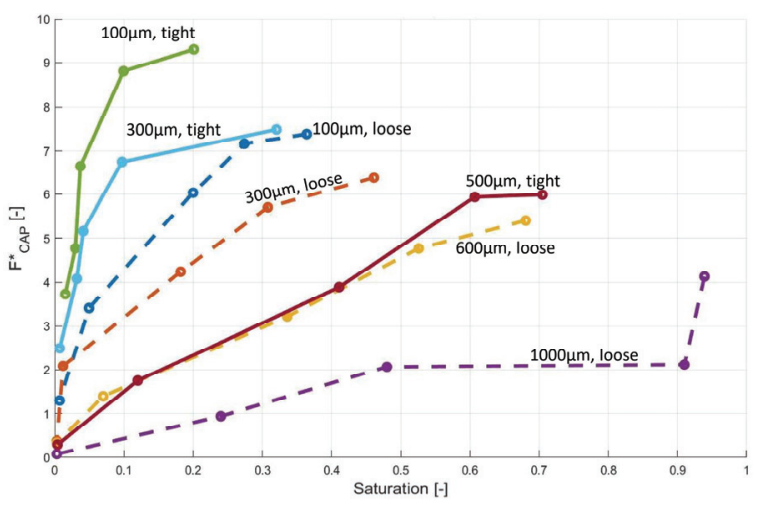

Fig. 7. The value of (non-dimensional) intergranular (adhesion) force at its maximum, and at the onset of first, second, third and fourth consecutive jump (snap through) at the air entry for five-grain clusters, loose (solid line) and tight (dashed line).

The first instability and the air entry occurs relatively soon after the intergranular (adhesive) force maximum, and at the force only slightly below $(\sim 5 \%)$ the maximum. For both tight and loose systems, for small separations (lower porosity systems) the air entry requires a higher intergranular force, and it takes place when relatively lot of the initial water has evaporated. The larger the gap between the grains, the lower the intergranular force at the air entry (and in general) and the smaller the amount fraction of water that has evaporated at the air entry.

For larger separations (loose clusters) simulating higher porosity medium, the first instability and air entry occurs after between only $9 \%$ or $72 \%$ of water has evaporated from the cluster. For the smallest separations (tight clusters) the first air entry takes place after already $40-90 \%$ water has evaporated. This means that for loose soils the air would start to penetrate soil so much earlier in the process than tight ones. As the media considered here are not deformable, that signifies that for loose systems the mechanism of desaturation through instabilities (air entry) kicks in much earlier than for the tight systems. For tight systems, the water loss occurs mainly via slow surface evaporation over a large part of duration of the process, without much of instabilities until very late. A similar trend is seen for smaller assembles, i.e. of 4 and 3 grains [5]. Fig. 8 shows the maximum adhesion force for 3-and 4- grain systems against cluster saturation for different separations, based on results presented by [5].

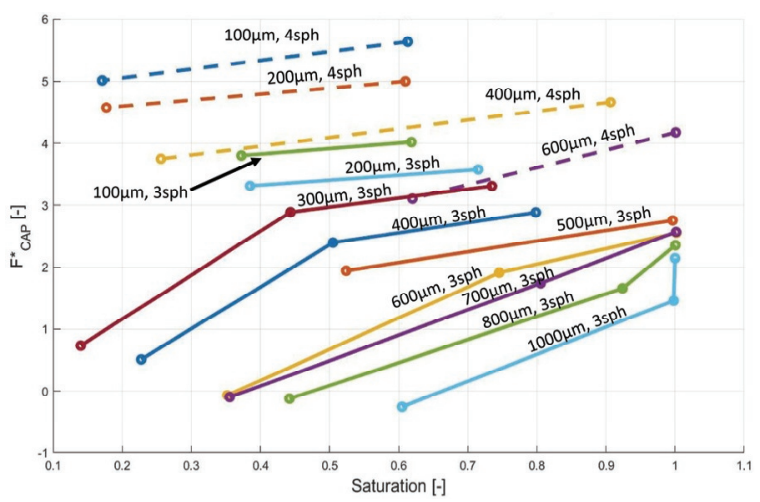

Fig. 8. The value of (non-dimensional) intergranular (adhesion) force at its maximum, and at the onset of first, and second, jump (snap through) at the air entry for three- (solid line) and four-grain (dashed line) clusters.

\subsection{Discussion 2}

The focus in unsaturated soil mechanics is on the air entry understood as "the first air entry", which is considered as equivalent to the onset of desaturation, and attributed with the corresponding pressure as "air entry pressure", as a property of the given soil. However, as the evaporation continues, the water/air boundary evolves, and subsequent instabilities and air entries take place at values of the intergranular force each time smaller, with the biggest values at smallest separations, see Figs. 7 and 8, where data both for tight and loose clusters are shown. The observed force decrease is consistent with other cases (two-grain bridges, [2, 4]), with the intergranular force invariably decreasing to zero). Each air entry is synonymous to a intergranular 
adhesion force drop, roughly over the same order of the magnitude of about $2 \times 10^{-4} \mathrm{~N}$, which is about $20 \%$ of the maximum force for the loose systems, and about $12.5 \%$ for tighter systems (see Fig. 5). Interestingly, the amount of the drop is practically the same for both modes of air entry: the meniscus (saddle) instability and a thin-sheet (double bowl) instability.

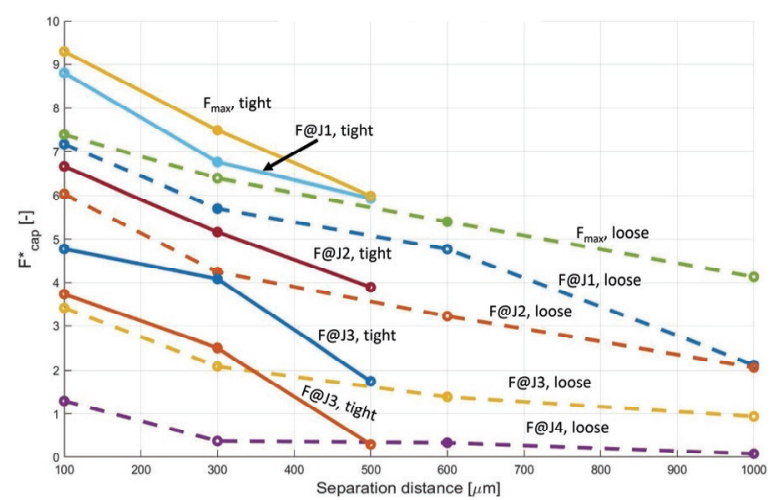

Fig. 9. The value of (non-dimensional) intergranular adhesion force at its maximum $\left(\mathrm{F}_{\max }\right)$, and at the onset of the first, $\mathrm{F}_{(\mathrm{J} 1}$, up to the fourth $\mathrm{F}_{@} \mathrm{~J} 4$ jump at the air entry for five-grain clusters, for different separations between grains: loose (solid-) and tight (dashed-line) clusters.

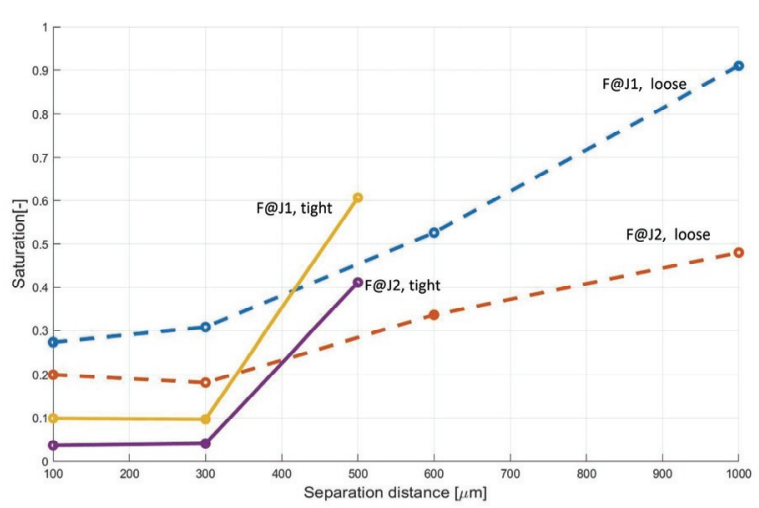

Fig. 10. The value of a critical cluster saturation at the onset of the first adhesion force jump at the first air entry for 3-, 4- and 5 -grain clusters, for different separation between grains.

Figure 9 shows that the value of the intergranular adhesion force at the first air entry for a 5 -grain cluster results to be smaller when the separations between grains are smaller, for both tight and loose clusters. The same is seen for subsequent jumps and for comparison for the maximum force in the drying process.

A similar conclusion may be reached for the adhesion force at the air entries for smaller clusters of 3 and 4 grains presented above in Fig. 8. Both graphs suggest that for materials with lower porosity their onset of desaturation with an air entry would occur at a higher adhesion force. By the same token (Fig. 10), the higher the separation between the grains (hence, the looser the soil), the higher the critical saturation, or a wetter the soil, at which in the process of drying a given cluster will undergo the first air entry, and hence will potentially crack, if constrained, following the observation of Hueckel et al. [1].

\section{Concluding remarks}

The above findings offer an alternative perspective on the interpretations of the air entry. First, the concept of the air entry pressure as an independent and its sole criterion (marker) clearly ignores the role of the surface tension force in the entry instability event (as more widely discussed by Hueckel et al. [5]). Second, the experiments clearly show a repetition of the unstable snap-throughs time and time again, progressively at a lower value of the intergranular adhesion force, and presumably of the associated pressure.

In general, the three modes of evolution of water configuration mentioned earlier, depend on the mode of air entry into the water body. The air entry mode is different for positive (double bowl), negative (or saddle) and zero (flat, at least in one cross-section) Gaussian curvature (GC) points. GC is the product of the two principal curvatures, of the water body surface, as illustrated in Fig. 2.

Currently, with the imaging techniques employed, we are not able to provide a numerical value of the $\mathrm{GC}$, and are not in the position to advance a hypothesis about quantitative criteria for the air entry, that is likely to be determined at a lower scale than discussed here.

From a phenomenological point of view it is concluded that the higher the separation between the grains or, the higher the soil porosity, the higher the critical cluster saturation, or in general, the wetter the soil, at which in the process of drying the first air entry will take place, and hence the soil will potentially crack, if constrained, following the observation of Hueckel et al. [1]. The results presented are valid for granular systems, and their generalization for clays requires to examine the mechanisms of evaporation and air entry in the presence of adsorbed water.

The authors acknowledge the support of US DOE grant DENE0008746.

\section{References}

1. T. Hueckel, B. Mielniczuk, M.S. El Youssoufi, L. B. $\mathrm{Hu}, \mathrm{L}$. Laloui, A three-scale cracking criterion for drying soils, Acta Geophys. 62, 1049 (2014), https://doi.org/10.2478/s11600-014-0214-9

2. B. Mielniczuk, T. Hueckel, M.S. El Youssoufi, Evaporation-induced evolution of the capillary force between two grains, Granular Matter 16, 815 (2014), DOI: $10.1007 / \mathrm{s} 10035-014-0512-6$

3. B. Mielniczuk, M.S. El Youssoufi, L. Sabatier, T. Hueckel, Rupture of an evaporating liquid bridge between two grains, Acta Geophysica 62, 1087 (2014), https://doi.org/10.2478/s11600-014-0225-6

4. B. Mielniczuk, T. Hueckel, M.S. El Youssoufi, Laplace pressure evolution and four instabilities in evaporating two-grain liquid bridges, Powder Technol. 283, $137 \quad$ (2015) https://doi.org/10.1016/j.powtec.2015.05.024

5. T. Hueckel, B. Mielniczuk, M.S. El Youssoufi, Adhesion-force micro-scale study of desiccating 
granular material, Geotechnique (2019), https://doi.org/10.1680/jgeot.18.P.298

6. G.I. Taylor and D. H. Michael, On making holes in a sheet of fluid, J. Fluid Mechanics 58, 625 (1973)

7. A.M. Anderson, L.N. Brush and S.H. Davis, Foam mechanics: spontaneous rupture of thinning liquid films with Plateau borders, J. Fluid Mechanics, 658, 63 (2010), DOI: 10.1017/S0022112010001527

8. C. Ma, T. Hueckel, Can. Geo. J. 29, 1087 (1992), https://doi.org/10.1139/t92-125

9. J.C. Fernandez-Toledano, T.D. Blake, P. Lambert, J. De Coninck, On the Cohesion of Fluids and Their Adhesion to Solids: Young's Equation at the Atomic Scale, Adv. Colloid Interface Sci. 245, 102 (2017)

10. J. Bear, Dynamics of Fluids in Porous Media. (Elsevier, New York, 1972)

11. N. Lu, W.J. Likos, Unsaturated soil mechanics (John Wiley \& Sons., Hoboken, NJ, USA, 2004)

12. R.T. Armstrong, N. Evseev, D. Koroteev and S. Berg, Modeling the velocity field during Haines jumps in porous media, Adv. Wat. Resour. 77, 57 (2015) 